\title{
A NEW CRITERION FOR CLOSE-TO-CONVEXITY OF PARTIAL SUMS OF CERTAIN HYPERGEOMETRIC FUNCTIONS ${ }^{1}$
}

\author{
MASSOUD JAHANGIRI \\ Kent State University, Department of Mathematics \\ Burton, Ohio 44021 USA
}

(Received February, 1996; Revised January, 1997)

\begin{abstract}
We consider the partial sums of certain hypergeometric functions and establish conditions imposed on the locations of zeros of those polynomials in order to be close-to-convex in the open unit disk.

Key words: Gaussian Hypergeometric Functions, Convolutions, Convex, Starlike, and Close-to-Convex Functions.
\end{abstract}

AMS subject classifications: $33 \mathrm{C} 05,30 \mathrm{C} 45,30 \mathrm{C} 50$.

\section{Introduction}

Let $\alpha_{i} \quad(i=1,2, \ldots p)$ and $\beta_{j}(j=1,2, \ldots, q)$ be complex numbers with $\beta_{j} \neq 0,-1$, $-2, \ldots ; j=1,2, \ldots, q$. The generalized hypergeometric function ${ }_{p} F_{q}(z)$ is defined by

$$
{ }_{p} F_{q}(z)={ }_{p} F_{q}\left(\alpha_{1}, \ldots, \alpha_{p} ; \beta_{1}, \ldots, \beta_{q} ; z\right)=\sum_{n=0}^{\infty} \frac{\left(\alpha_{1}\right)_{n} \ldots\left(\alpha_{p}\right)_{n}}{\left(\beta_{1}\right)_{n} \ldots\left(\beta_{q}\right)_{n}} \cdot \frac{z^{n}}{n !},
$$

where $p \leq q+1,(\lambda)_{0}=1$, and $(\lambda)_{n}=\frac{\Gamma(\lambda+n)}{\Gamma(\lambda)}=\lambda(\lambda+1) \ldots(\lambda+n-1)$ if $n=1,2, \ldots$ The series given by (1) converges absolutely for $|z|<\infty$ if $p<q+1$, and for $z$ in the open unit disk $U=\{z:|z|<1\}$ if $p=q+1$. For suitable values of $\alpha_{i}$ and $\beta_{j}$, ${ }_{p} F_{q}(z)$ is closely related to classes of analytic and univalent functions. A quote from Miller and Mocanu [12] reads: "The surprising use of hypergeometric functions in the recent proof of the Bieberbach conjecture by L. de Branges [4] in 1985 has prompted renewed interest in these classes of functions. Prior to this proof, there had been only a few articles in the literature dealing with the relationship between these special functions and univalent function theory." It is well-known that hypergeometric and univalent functions play important roles in a large variety of problems encountered in applied mathematics, probability and statistics, operations research, signal theory, moment problems, and other areas. For further references and applications see Exton $[6,7]$ and Rönning [16]. In this paper we introduce a new approach for studying the relationships between classes of hypergeometric and analytic univalent functions. We

\footnotetext{
${ }^{1}$ This work was supported by the Kent State University Research Council.
} 
hope this approach will motivate further work in this direction. In Section 2 we discuss the convolution properties of classes of hypergeometric functions ${ }_{2} F_{1}$. In Section 3 we determine the conditions on the location of the zeros of the partial sums of ${ }_{2} F_{1}$ that are close-to-convex of order $\alpha ; \alpha>0$.

\section{Convolution Properties of ${ }_{2} F_{1}$}

For $p=q+1=2$, the series defined by (1) gives rise to the Gaussian hypergeometric series ${ }_{2} F_{1}(a, b: c: z)$. This reduces to the elementary Gaussian geometric series $1+$ $z+z^{2}+\ldots$ if $(i) a=c$ and $b=1$ or $(i i) a=1$ and $b=c$. When $\operatorname{Re}(c)>\operatorname{Re}(b)>0$, we obtain

$$
{ }_{2} F_{1}(a, b ; c ; z)=\frac{\Gamma(c)}{\Gamma(b) \Gamma(c-b)} \int_{0}^{1} \frac{t^{b-1}(1-t)^{c-b-1}}{(1-t z)^{a}} d t .
$$

As a special case, we see that

and

$$
{ }_{2} F_{1}(1,1 ; a ; z)=(a-1) \int_{0}^{1} \frac{(1-t)^{a-2}}{1-t z} d t
$$

$$
{ }_{2} F_{1}(a, 1 ; 1 ; z)=\frac{1}{(1-z)^{a}}
$$

so that

$$
{ }_{2} F_{1}(1,1 ; a ; z) *{ }_{2} F_{1}(a, 1 ; 1 ; z)=\frac{1}{1-z}={ }_{2} F_{1}(1,1 ; 1 ; z) \text {. }
$$

The operator $*$ stands for the Hadamard product or convolution of two power series $f(z)=\sum_{n=0}^{\infty} a_{n} z^{n}$ and $g(z)=\sum_{n=0}^{\infty} b_{n} z^{n}, \quad$ that is, $(f * g)(z)=f(z) * g(z)=$ $\sum_{n=0}^{\infty} a_{n} b_{n} z^{n}$. If $f$ and $g$ are analytic in $U$ then the convolution $f * g$ is also analytic in $U$. An alternative representation for the Hadamard product is the convolution integral

$$
(f * g)(z)=\frac{1}{2 \pi i} \int_{|\zeta|=1} \zeta^{-1} f\left(\frac{z}{\zeta}\right) g(\zeta) d \zeta,|z|<1 .
$$

Two power series $f$ and $g$ are said to be the convolution inverses of each other whenever the convolution $f * g$ gives the identity power series $1 /(1-z)$. In this case we write $f=g^{(-1)}$ or $g=f^{(-1)}$ So, ${ }_{2} F_{1}(1,1 ; a ; z)$ and ${ }_{2} F_{1}(a, 1 ; 1 ; z)$ are convolution inverses of each other. The function $z_{2} F_{1}(1,1 ; 1 ; z)=z /(1-z)$ is a typical example of a convex univalent function. A function $f(z)$ which is analytic in $U$ is said to be convex univalent in $U$ [5] if $f(z)$ is univalent and conformally maps the disk $\{z$ : $|z|<r<1\}$ onto a convex region so that the boundary of the region is a simple closed convex curve. Alexander [3] showed that $f(z)$ is convex in $U$ if and only if $z f^{\prime}(z)$ is starlike in $U$. A function $f(z)$ is said to be starlike in $U$ [5] if every point of the image of $\{z:|z|=r<1\}$ under the conformal mapping $f$ is "visible" from the origin. A necessary and sufficient condition for $f(z)$ to be analytic and starlike of order $\alpha ; \alpha \leq 1$ in $U$ is that $\operatorname{Re}\left\{\frac{z f^{\prime}(z)}{f(z)}\right\} \geq \alpha, z \in U$. Let $S^{*}(\alpha)$ denote the class of func- 
tions $f$ that are analytic and starlike of order $\alpha ; \alpha \leq 1$ in $U$. With a simple calculation [17] we see that if $f \in S^{*}(\alpha), \alpha \leq 1$, then there exists a probability measure $\mu$ on $\partial U$ such that

In particular,

$$
f(z)=\int_{\partial U} z_{2} F_{1}(2-2 \alpha, 1 ; 1 ; z \zeta) d \mu(\zeta) .
$$

$$
f(z) \ll z_{2} F_{1}(2-2 \alpha, 1 ; z)=\frac{z}{(1-z)^{2-2 \alpha}} .
$$

For the power series $f(z)=\sum_{n=0}^{\infty} a_{n} z^{n}$ and $F(z)=\sum_{n=0}^{\infty} A_{n} z^{n}$ convergent in $U$ we say $f(z)$ is dominated or marjorized by $F(z)$, in notation, $f(z) \ll F(z)$, if $\left|a_{n}\right| \leq A_{n}$.

For $t>-1 / 2, k>-1 / 2$, and $|x| \leq 1$ we define $F(t, k, x)$ by

$$
F(t, k, x)=\sum_{n=0}^{\infty} \frac{P_{n}^{(t, k)}(x)}{P_{n}^{(t, k)}(1)} z^{n+1}
$$

where $z \in U$, and $P_{n}^{(t, k)}(x)$ are (see Lewis [11]) the Jacobi polynomials

$$
P_{n}^{(t, k)}(x)=\frac{(1+t) n}{n !}{ }_{2} F_{1}\left(-n, t+k+n+1 ; t+1 ; \frac{1-x}{2}\right) .
$$

From the definitions of $F(t, k, x)$, convex, and starlike functions, it follows that $F\left(\frac{1}{2}, \frac{1}{2}, x\right)$ is convex in $U$ and $F(0,0, x)$ is starlike of order $1 / 2$ in $U$. Next, we use starlike functions to construct a class of analytic functions which is the subject to our investigation in the following section. We say a function $f(z)$ is analytic and close-toconvex (or linearly accessible) in $U$ [5] if the "complement" of the image of $\{z:|z| \leq r<1\}$ under conformal mapping $f$ is the union of a family of non-intersecting half-lines. A function $f(z)$ is said to be analytic and close-to-convex of order $\alpha$; $\alpha>0$ in $U$, in notation $C(\alpha)$, if and only if there exists a function $g$ in $S^{*}(0)$ such that

$$
\left|\arg \frac{z f^{\prime}(z)}{g(z)}\right|<\frac{\alpha \pi}{2}, \quad z \in U .
$$

Many authors including ([1, 2 and 10-18]) studied the properties of classes of convex, starlike, and close-to-convex hypergeometric functions. In this paper we investigate the locations of the zeros of partial sums of close-to-convex hypergeometric series. We are not aware of any previous work that has adopted this approach.

\section{Partial Sums of ${ }_{2} F_{1}$}

Let ${ }_{2} F_{1}(a, b ; c ; z)$ be so that its $n$-th partial sums ${ }_{2} F_{1}(a, b ; c ; z)_{n}$ can be written as

$$
{ }_{2} F_{1}(a, b ; c ; z)_{n}=\prod_{k=1}^{n}\left(1+z e^{i \phi_{k}}\right),
$$

where $0 \leq \phi_{1} \leq \phi_{2} \leq \ldots \leq \phi_{n} \leq \phi_{1}+2 \pi$ and $z \in U$. For such polynomials we have

$$
z_{3} F_{2}(a, b, 1 ; c, 2 ; z)_{n}=\int_{0}^{z}{ }_{2} F_{1}(a, b ; c ; \zeta)_{n} d \zeta \text {. }
$$


Actually, this is true even without truncating the Gaussian hypergeometric series and holds also true under certain convergence conditions for the infinite generalized hypergeometric series defined by (1).

Using the change of argument properties of close-to-convex functions in conjunction with a result due to the author (let $\beta=2+\alpha$ in [8]) we have the following theorem.

Theorem 1: Let $1 \leq \alpha \leq n$. Then $z_{3} F_{2}(a, b, 1 ; c, 2 ; z)_{n}$ is close-to-convex of order $\alpha: \alpha>0$, if and only if

$$
\max \left\{0, \frac{2(m+1-\alpha) \pi}{n+2}\right\} \leq \phi_{l+m}-\phi_{l} \leq \min \left\{\frac{2(m+1+\alpha) \pi}{n+2}, 2 \pi\right\},
$$

where $2 \leq l+m \leq n$ and $1 \leq l, m \leq n-1$.

To see the relations between the parameters $a, b, c$, and $\phi_{k}$ we examine a special case when $\alpha=1$ and $n=2$. For $\alpha=1$, inequality (3) reduces to

$$
\frac{2 m \pi}{n+2} \leq \phi_{l+m}-\phi_{l} \leq \frac{2(m+2) \pi}{n+2}
$$

Consequently, for $n=2$ we deduce that if $z_{3} F_{2}(a, b, 1 ; c, 2 ; z)_{2}$ is in $C(1)$, then

$$
{ }_{2} F_{1}(a, b ; c ; z)_{2} \ll 1+\sqrt{2} z+z^{2} \text {. }
$$

An extremal case, which satisfies condition (5), is

$$
{ }_{2} F_{1}\left(a, \frac{\sqrt{2}-2-2 a}{a+2} ; \frac{a(\sqrt{2}-2-2 a)}{(a+2) \sqrt{2}} ; z\right) \text {. }
$$

For a non-extremal case, let $a=\frac{1}{2}, b=2$, and $c=\frac{-1+\sqrt{10}}{2}$. Therefore,

$$
\left.{ }_{2} F_{1}\left(\frac{1}{2}, 2 ; \frac{1}{2}(-1+\sqrt{10}) ; z\right)\right)_{2}=\prod_{k=1}^{2}\left(1+z e^{i \phi_{k}}\right) \text {. }
$$

In this case, $\phi_{1}=\cos ^{-1}\left(\frac{1+\sqrt{10}}{2}\right)$ and $\phi_{2}=2 \pi-\phi_{1}$, which satisfies condition (4) when $n=2$. As a more general case, let $2 \leq \alpha \leq n=4$. A necessary condition for $z_{3} F_{2}(a, b, 1 ; c, 2 ; z)_{4}$ to be close-to-convex of order $\bar{\alpha}$ is that

$$
\left.{ }_{2} F_{1}(a, b ; c ; z)\right)_{4} \ll P_{4}(z ; \alpha)=(1+z)^{\alpha-1} \prod_{j=1}^{5-\alpha}\left(1+z e^{\frac{(2 j+\alpha-6) \pi i}{6}}\right) .
$$

The polynomial $P_{4}(z ; \alpha)$ plays an important role in the convolution of close-to-convex hypergeometric functions. The following theorem is a consequence of the above argument and an application of a result due to the author [9].

Theorem 2: If $z_{3} F_{2}\left(a_{1}, b_{1}, 1 ; c_{1}, 2 ; z\right)_{4}$ and $z_{3} F_{2}\left(a_{2}, b_{2}, 1 ; c_{2}, 2 ; z\right)_{4}$ are close-toconvex of order $\alpha$; $\alpha=2,3$ and 4 , then the convolution $z\left\{{ }_{5} F_{3}\left(a_{1}, a_{2}, b_{1}, b_{2}, 1\right.\right.$; $\left.\left.c_{1}, c_{2}, 2 ; z\right)_{4} * P_{4}(z ; \alpha)\right\}$ has the same property.

The above theorem for the case $\alpha=1$ was proved by Suffridge ([19], Theorem 5). We do not know if a similar convolution invariance property holds for the general case $\alpha>0$. This remains open. A potential candidate for further investigation is the extremal polynomial 


$$
P_{n}(z ; \alpha)=(1+z)^{[\alpha]-1} \prod_{j=1}^{n+1-[\alpha]}\left(1+z e^{\frac{(2 j+[\alpha]-n-2) \pi i}{n+2}}\right)
$$

where $[\alpha]$ stands for the integer part of $\alpha$. (See also the Conjecture in [9].)

\section{References}

[1] Ahuja, O.P. and Jahangiri, M., Inclusion relations between classes of hypergeometric functions, J. Appl. Math. Stoch. Anal. 7:1 (1994), 79-89.

[2] Ahuja, O.P. and Silverman, H., Function classes related to Ruscheweyh derivatives, J. Aust. Math. Soc. 47 (1989), 438-444.

[3] Alexander II, J.W., Functions which map the interior of the unit circle upon simple regions, Annals Math. 17 (1915), 12-22.

[4] de Branges, L., A proof of the Bieberbach conjecture, Acta Math. 154 (1985), 137-152.

[5] Duren, P.L, Univalent Functions, Springer-Verlag, New York 1983.

[6] Exton, H., Multiple Hypergeometric Functions and Applications, Ellis Horwood Ltd. (Chichester), John Wiley and Sons (Halsted Press), London, New York, Sydney, Toronto 1976.

[7] Exton, H., Handbook of Hypergeometric Integrals: Theory, Applications, Tables, Computer Programs, Ellis Horwood Ltd. (Chichester), John Wiley and Sons (Halsted Press), London, New York, Sydney, Toronto 1978.

[8] Jahangiri, M., A gap condition for the zeros of certain polynomials in Kaplan classes $K(\alpha, \beta)$, Mathematika 34 (1987), 53-63.

[9] Jahangiri, M., Weighted convolutions of certain polynomials, Bull. Aust. Math. Soc. 40 (1989), 397-405.

[10] Jahangiri, M. and Silvia, E.M., Some inequalities involving generalized hypergeometric functions, Univalent Functions, Fractional Calculus, and their Applications (ed. by H.M. Srivastava and S. Owa), Ellis Horwood Ltd. (Chichester), John Wiley and Sons (Halsted Press), Chichester, Brisbane, New York, Toronto (1989), 65-74.

[11] Lewis, J.L., Applications of a convolution theorem to Jacobi polynomials, SIAM J. Math. Anal. 10 (1979), 1110-1120.

[12] Miller, S.S. and Mocanu, P.T., Univalence of Gaussian and confluent hypergeometric functions, Proc. Amer. Math. Soc. 110:2 (1990), 333-342.

[13] Noor, K.I., Some results on certain subclasses of analytic functions involving generalized hypergeometric functions and Hadamard product, Int. J. Math. \& Math. Sci. 15:1 (1992), 143-148.

[14] Noor, K.I., On certain starlike and convex hypergeometric functions, Panam. Math. J. 4 (1994), 29-34.

[15] Owa, S. and Srivastava, H.M., Univalent and starlike generalized hypergeometric functions, Canad. J. Math. 39 (1987), 1057-1077.

[16] Rönning, F., PC-fractions and Szego polynomials associated with starlike univalent functions, Numerical Algorithms 3 (1992), 383-392.

[17] Ruscheweyh, St., Convolutions in geometric function theory, Seminaire de Math. Sup., Les Press de L' universite de Montreal 1982.

[18] Ruscheweyh, St. and Singh, V., On the order of starlikeness of hypergeometric functions, J. Math. Ana. Appl. 113 (1986), 1-11. 
[19] Suffridge, T.J., Starlike functions as limits of polynomials, Adv. in Complex Function Theory, Lecture Notes in Math 505, Springer-Verlag, New York (1973/74), 164-203. 


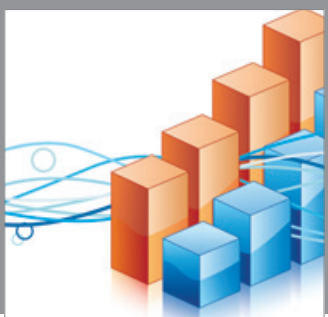

Advances in

Operations Research

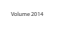

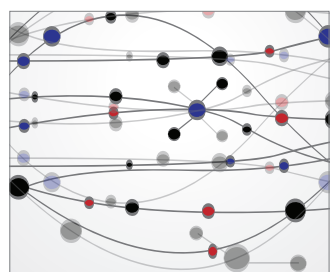

\section{The Scientific} World Journal
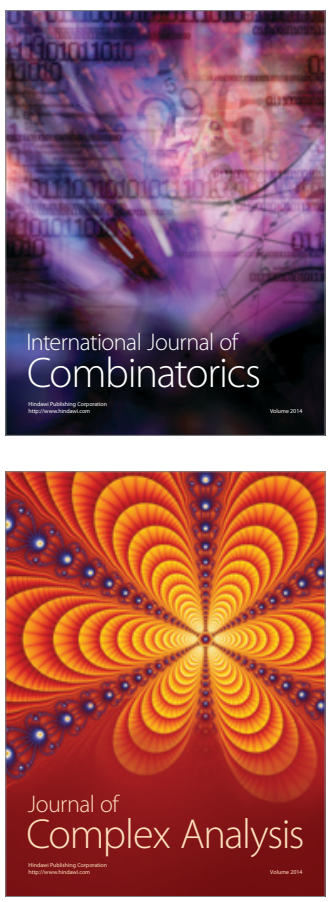

International Journal of

Mathematics and

Mathematical

Sciences
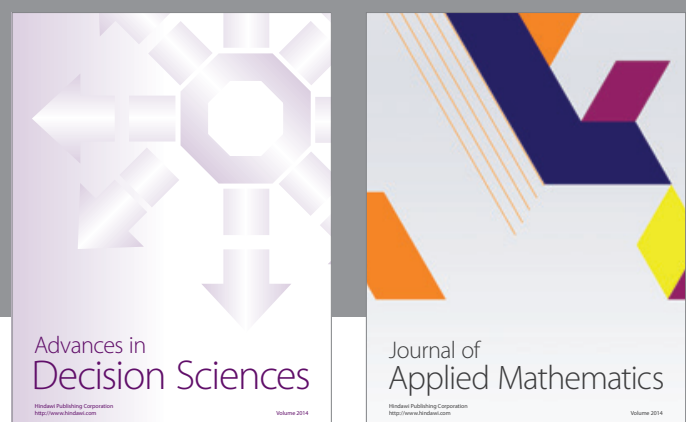

Journal of

Applied Mathematics
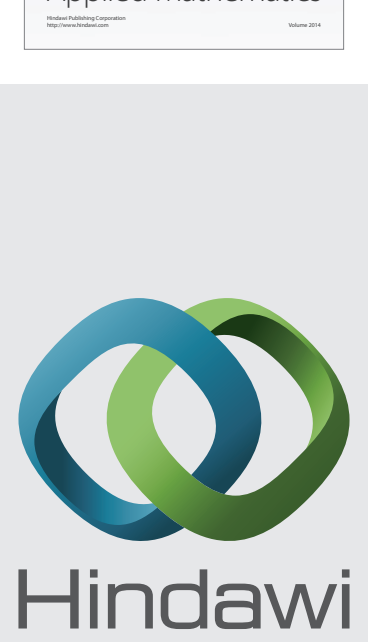

Submit your manuscripts at http://www.hindawi.com
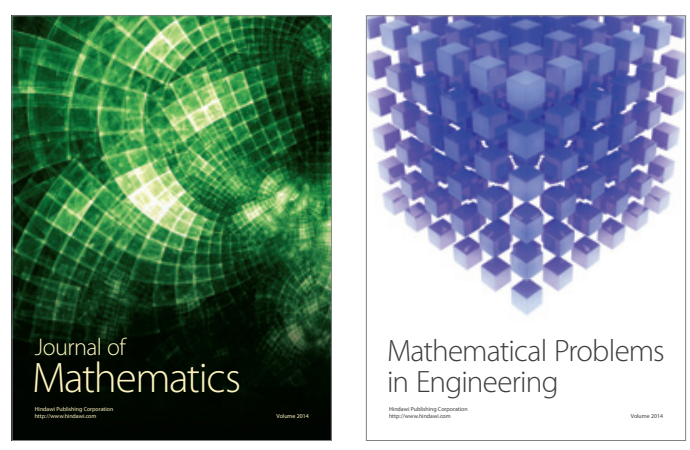

Mathematical Problems in Engineering
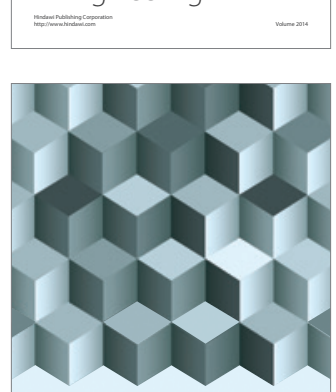

Journal of

Function Spaces
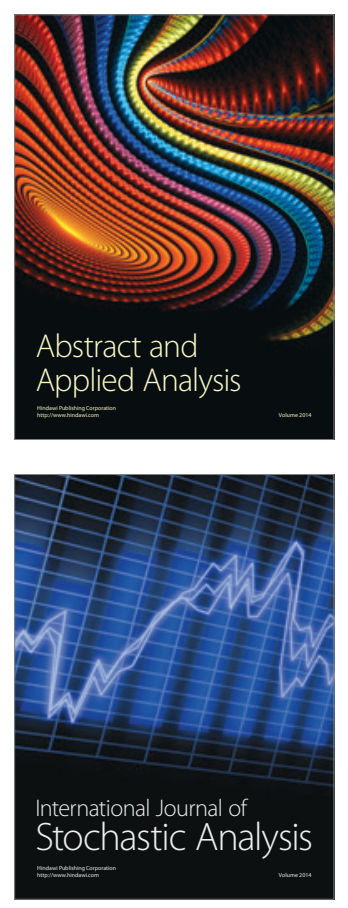

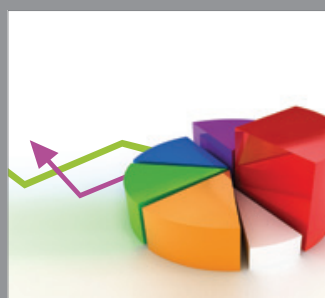

ournal of

Probability and Statistics

Promensencen
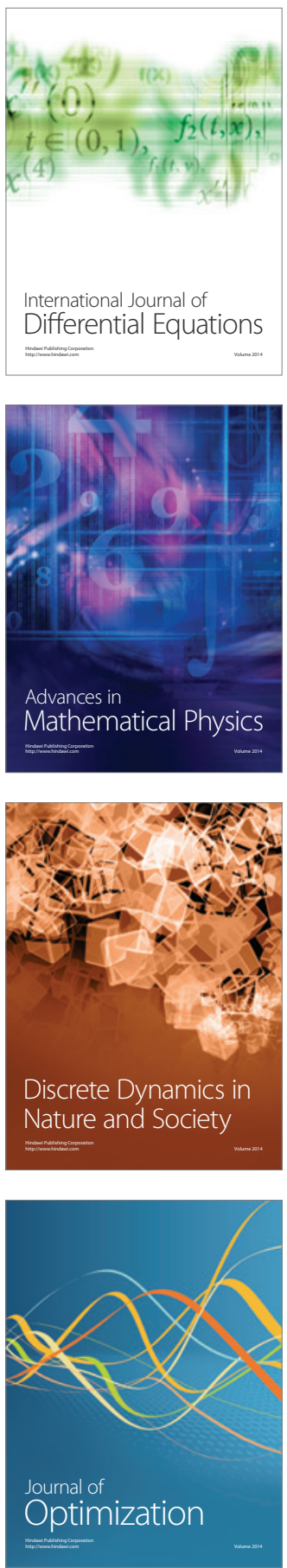